\title{
Characterization and Classification of Soils in the Central Parts of Prakasam District in Andhra Pradesh, India
}

\author{
Ch. Chandra Sekhar ${ }^{1 *}$, M.V.S. Naidu ${ }^{2}$, T. Ramprakash $^{1}$ and D. Balaguravaiah $^{1}$ \\ ${ }^{1}$ Department of Soil Science and Agricultural Chemistry, College of Agriculture, ANGRAU, \\ Hyderabad-500 030, Telangana, India \\ 2 Department of Soil Science and Agricultural Chemistry, S.V. Agricultural College, ANGRAU, \\ Tirupati-517 502, A.P., India \\ *Corresponding author
}

\begin{tabular}{|c|c|}
\hline & A B S T R A C T \\
\hline & $\begin{array}{l}\text { Seven typical pedons from Gundlasamudram, Vallaipalem, Peddaalavalapadu, } \\
\text { Vijayalaxmipuram, Rajupalem, Gangapalem and Bakkireddipalem villages representing } \\
\text { the major land forms in the central parts of Prakasam district of Andhra Pradesh viz., } \\
\text { uplands and plains, developed from granite-gneiss parent material under varying land use }\end{array}$ \\
\hline Keywords & $\begin{array}{l}\text { were studied for their morphological characteristics, physical and physico-chemical } \\
\text { properties, soil genesis and nutrient status. Soils were shallow to very deep, slightly }\end{array}$ \\
\hline $\begin{array}{l}\text { Soil classification, } \\
\text { Soil survey, Cambic } \\
\text { horizon, } \\
\text { Slickensides, } \\
\text { Pressurefaces and } \\
\text { Soil fertility. }\end{array}$ & $\begin{array}{l}\text { alkaline to alkali ( } \mathrm{pH} 7.63 \text { to } 10.48 \text { ) in reaction, non-saline and had iso-hyperthermic } \\
\text { temperature and ustic soil moisture regimes. Texture, organic carbon, CEC and base } \\
\text { saturation in the profiles ranged from sandy loam to clay, } 0.14 \text { to } 0.72 \text { per cent, } 9.14 \text { to } \\
53.85 \mathrm{cmol}\left(\mathrm{p}^{+}\right) \mathrm{kg}^{-1} \text { and } 61.95 \text { to } 92.78 \text { per cent, respectively. Soils were low in available } \\
\text { nitrogen, medium in available phosphorus, medium to high in available potassium and } \\
\text { high in available sulphur (except only one sample). However, the soils were sufficient in }\end{array}$ \\
\hline Article Info & the pedons 2 and 4 and in the \\
\hline $\begin{array}{l}\text { Accepted: } \\
23 \text { September } 2017 \\
\text { Available Online: } \\
10 \text { October } 2017\end{array}$ & $\begin{array}{l}\text { second horizon of pedon 1) Cu and Mn. Pedons } 3 \text { and } 6 \text { were grouped under Entisols due } \\
\text { to absence of sub-surface diagnostic horizon and were classified as Lithic Ustorthent, } \\
\text { whereas pedons } 1,4,5 \text { and } 7 \text { were placed under Inceptisols due to presence of cambic } \\
\text { (Bw) sub-surface diagnostic horizon and classified as Typic Haplustepts. Due to the }\end{array}$ \\
\hline & $\begin{array}{l}\text { presence of vertic features like slickensides, pressurefaces, cracks and presence of more } \\
\text { than } 30 \% \text { clay in all the horizons, the pedon } 2 \text { was grouped under Vertisols and classified } \\
\text { as Typic Haplusterts. Recommendations were made based on the soil fertility for better } \\
\text { crop production without deteriorating the soil health. }\end{array}$ \\
\hline
\end{tabular}

\section{Introduction}

Soil is one of the most important natural resources for existence of living creatures. Yet, it is non-renewable and finite natural resource. Indiscriminate use of soil coupled with lack of proper management has led to its degradation echoing the concerns of the planners, researchers and farmers (Sharma, 2006). This calls for a scientific approach in studying, management and development of this natural resource at various levels. Soil 
resource inventory provides an insight into the potentialities and limitations for its best management. It also provides adequate information in terms of land forms, natural vegetation as well as characteristics of soils which can be utilized for land resources management and development (Manchanda et al., 2002). Rational utilization of land resources can be achieved by optimizing its use, which demands evaluation of land for alternative land use. Characterization, classification and evaluation of soils under different land uses are the first milestone in developing sustainable and eco-friendly land use models. No Information is available especially on characterization, classification and genesis of soils pertaining to Prakasam district in general and in the central parts of the district in particular. Hence, present study was taken up to characterize, classify and evaluate the soils in central parts of Prakasam district.

\section{Materials and Methods}

\section{Study area}

The study area is located between $14^{\circ} 57^{\prime}$ and $16^{\circ} 17^{\prime}$ North latitudes and $78^{\circ} 43^{\prime}$ and $80^{\circ}$ $25^{\prime}$ East longitudes with an altitude ranging from 50 to $94 \mathrm{~m}$ (ms). Soils in the central parts of Prakasam district have been mainly developed from granite-gneiss parent materials with calcareousness in some profiles (Table 1). The area is classified under semiarid monsoonic climate with distinct summer, winter and rainy seasons. The area receives a mean annual precipitation of $747 \mathrm{~mm}$. The mean annual temperature of the study area was $29.49^{\circ} \mathrm{C}$, with a mean summer temperature of $32.33^{\circ} \mathrm{C}$ and mean winter temperature of $26.09^{\circ} \mathrm{C}$. The maximum temperature recorded in the study area in the last 10 years is $44.60^{\circ} \mathrm{C}$, in the month of May and the minimum temperature recorded is $20.36^{\circ} \mathrm{C}$, in the month of January. The soil moisture regime (SMR) has been computed as ustic and the soil temperature regime (STR) as iso-hyperthermic. The natural vegetation observed in this area comprises of species like Acacia nilotica, Borassus flabellifer, Parthenium hysterophorus, Calotropis gigantia, Prosopis juliflora, Tamarindus indica, Azadirachta indica, Cassia auriculata, Ziziphus mauritiana and Cyperus rotundus etc.

\section{Methodology}

A reconnaissance soil survey was conducted in Prakasam district using toposheets with 1:50,000 scale as per procedure outlined by AIS \& LUS (1970). Auger bores, minipots, road cuts and 12 pedons located on plains and uplands were studied. Seven typical pedons from soil correlation exercise were selected from the study area in the central parts of Prakasam district. These seven typical pedons were studied in detail for their morphological properties (Table 2) in the field as per the procedure outlined in the Soil Survey Manual (Soil Survey Division Staff, 2000). Based on the field observations, horizon-wise soil samples were collected from the selected profiles in the study area and were analyzed for their physical, physico-chemical properties and available major and micro nutrient status using standard procedures. The soils were then classified taxonomically following their characteristics observed from the field and laboratory studies (Soil Survey Staff, 2014).

\section{Results and Discussion}

\section{Soil morphology}

The soils in study area were found to be shallow to very deep with poorly to welldrained conditions. The distinctness of horizon boundaries was clear to gradual and their topography was smooth to wavy. Colour 
notations of studied profiles have shown hue ranging from $2.5 \mathrm{YR}$ to $10 \mathrm{YR}$. Profiles 1,2 and 6 have shown hue 10YR with values 3-4 and chrome of 2-4. Profiles 4 and 5 have shown hue 7.5YR with values 3-4 and chrome of 3-4. Profile 7 has hue of 5YR with values 3-4 and chrome of 3-4, where as profile 3 has hue of $2.5 \mathrm{YR}$ with values $3-4$ and chrome of 4-6.

The soil colour appears to be the function of chemical and mineralogical composition as well as textural make up of soils and conditioned by topographic position and moisture regime (Walia and Rao, 1997). Textural class of the soils varied from sandy loam to clay. This textural variation might be due to differences in composition of parent material, topography, in-situ weathering and translocation of clay by eluviation and age of soils (Geetha Sireesha and Naidu, 2013). The structure of the soils was sub-angular blocky and angular blocky. The blocky structure i.e., angular and sub-angular blocky were attributed to the presence of higher quantities of clay fraction (Sharma et al., 2004).

The consistence of the soils varied from soft to very hard (dry), friable to very firm (moist) and slightly sticky and slightly plastic to very sticky and very plastic (wet). Presence of sticky and plastic to very sticky and very plastic, firm to very firm and slightly hard to very hard consistence in wet, most and dry conditions, respectively may be due to high clay content of soil (Sarkar et al., 2001) and also due to dominance of smectite clay mineral (Leelavathi et al., 2010). Pedons 1, 2, 4, 5 and 7 exhibited a cambic (Bw) subsurface diagnostic horizon and pedon 2 had shown presence of Bss horizon also. However, pedons 3 and 6 did not have any diagnostic horizon. Strong to violent effervescence with dilute $\mathrm{HCl}$ was observed in all the pedons except in the surface horizon of pedon 3 .

\section{Soil characteristics}

\section{Physical characteristics}

Details of physical characteristics of soils in the study area are presented in table 3 . Particle size analysis data revealed that the clay content varied from 15.82 to 49.26 per cent. Increase in clay content with depth in all the pedons might be due to downward translocation of finer particles from the surface layers (Murthy, 1988). Silt content ranged from 6.85 to $23.32 \%$ and its content in general increased with depth. However, it exhibited an irregular trend with depth, in pedons 1 and 2, which might be due to variations in weathering of parent material or in situ formation (Satish Kumar and Naidu, 2012). Sand constituted the bulk of mechanical fractions (35.26-77.33\%), which indicates the siliceous nature of parent material.

Bulk density of different horizons of the pedons in the study area varied from 1.15 to $1.79 \mathrm{Mg} \mathrm{m}^{-3}$. Variations in bulk density of these soils were attributed to high amounts of expanding type clay minerals. Similar findings were also reported by Ram Prakash and Seshagiri Rao (2002). Bulk density of sub-surface horizons was higher than that of surface horizons and increased with depth, which was due to compaction of finer particles in deeper layers caused by the overhead weight of the surface layers (Thangasamy et al., 2005), high clay content in swelling clay soils (Ahuja et al., 1988) and lower organic matter and plant root concentration in the lower layers (Coughlan et al., 1986). The lower bulk density observed in the surface layers was due to continuous cultivation, high organic matter and higher biotic activities (Vara Prasad Rao et al., 2008). Particle density of soil horizons in the pedons of study area ranged between 2.35$2.68 \mathrm{Mg} \mathrm{m}^{-3}$. Particle density, in general, 
followed an increasing trend with depth, but was more or less uniform within the pedons (Gurumurthy et al., 1996). Water holding capacity of different pedons in the study area varied from 18.54 to 46.60 per cent. These variations were due to the differences in depth, clay, silt, sand and organic carbon contents. Low water holding capacity in sandy soils was due to high sand and less clay content which was also evident by the high significant and negative correlation $(\mathrm{r}=$ $0.806^{* *}$ ) found between water holding capacity and sand content. The irregular trend of water holding capacity with depth in pedons 1 and 2 was due to the eluviation and illuviation of finer fractions in different horizons.

\section{Physico-chemical characteristics}

Soils in the study area are slightly alkaline to alkali in nature with $\mathrm{pH}$ varying from 7.63 to 10.48. This was attributed to the nature of the parent material, leaching, presence of calcium carbonate and exchangeable sodium (Shalima Devi and Anil Kumar, 2010). All the pedons had shown low electrical conductivity values ranging from 0.04 to $1.38 \mathrm{dS} \mathrm{m}^{-1}$, indicating non-saline nature of these soils. The low electrical conductivity was due to free drainage conditions which favoured the removal of released bases by percolating and drainage water (Table 4).

Organic carbon content of soils in the study area was found to be low to medium and varied from 0.14 to 0.72 per cent (Table 4). The low carbon content in the soils might be due to the prevalence of tropical condition, which results in faster degradation of organic matter coupled with low vegetation cover, there by leaving less organic carbon in the soils (Nayak et al., 2002). The organic carbon content decreased with depth in all the pedons. Relatively high organic carbon content in the surface horizons is due to the addition of plant residues and farm yard manure to surface horizons (Ashokkumar and Jagdish Prasad, 2010).

CEC in pedons of the study area varied from

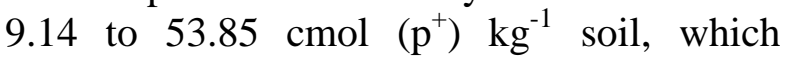
corresponds to clay content, type of clay mineral and organic carbon content present in these soils. Free $\mathrm{CaCO}_{3}$ ranged from 5.42 to 17.64 per cent in these soils and the higher $\mathrm{CaCO}_{3}$ content might be due to semi-arid climate which is responsible for the pedogenic processes resulting in the depletion of $\mathrm{Ca}^{+2}$ ions from the soil solution in the form of calcretes (Ashokkumar and Jagdish Prasad, 2010). The $\mathrm{CaCO}_{3}$ content increased with depth in all the pedons (except the lowest horizon in pedon 7) which was due to leaching of calcium and its subsequent precipitation due to high $\mathrm{pH}$ level. Pedon 7 showed an irregular distribution with depth, which is attributed to variable nature of geological material that contributed to these soils or rapid leaching of carbonates from the porous sandy horizons (Singh and Agrawal 2005).

Exchangeable bases in all pedons of the study area were in the order of $\mathrm{Ca}^{2+}>\mathrm{Mg}^{2+}>\mathrm{Na}^{+}>$ $\mathrm{K}^{+}$on the exchange complex. The percent base saturation (BSP) ranged from 61.95 to 92.78 per cent. The higher values of BSP observed is due to higher amount of $\mathrm{Ca}^{+2}$ ions occupying the exchange sites on the colloidal sites. The ratio between CEC and clay (CEC: clay) ranged from 0.28 to 1.22 and the CEC: clay ratio was used to identify the clay mineralogy (Ashokkumar and Jagdish Prasad, 2010).

\section{Soil genesis}

Soil profile examination in the study area showed distinctive horizontal layers, some of which were clearly visible. Significant changes were observed as these soils were 
developed from relatively unconsolidated parent material. Pedons 3 and 6 were developed from granite-gneiss; pedons 2, 4, 5 and 7 relatively more unconsolidated weathered granite-gneiss, whereas pedon 1 was developed from weathered calcareous gneiss parent material. High organic matter content was noticed due to accumulation of plant material and humus on the surface soils and to certain depth of sub-soil in all the pedons of the study area (Simonson, 1959). The surface horizon in all these pedons was dark in colour as compared to sub-surface horizons due to accumulation of organic matter.

Higher organic matter in the surface soils was due to continuous addition of organic matter through leaf fall, stubbles, roots and organic manures applied to the surface layers only (Bhaskar et al., 2004). Further, organic carbon was leached to lower layers also along with the percolating water (Leelavathi et al., 2009)

Soil formation was followed by translocation of material from one point to another within the soil. In this phase, eluviation and illuviation processes were of great significance. Development of B horizons in the pedons 1, 2, 4, 5 and 7 was the result of eluviation and illuviation processes within the profiles. Due to these processes the cambic horizon $(\mathrm{Bw})$ was formed. However, these processes were not operated in the pedons 3 and 6, thus no horizon development was observed in sub-surface layers of these pedons.

Soil forming processes like transformation of minerals and organic substances result in change in colour and structure in sub-soil leading to the development of cambic horizon $(\mathrm{Bw})$ in pedons $1,2,4,5$ and 7 . The study area has semi-arid monsoonic type of climate with high summer temperatures with scarce rainfall. Natural vegetation observed in the study area were perennial trees, annuals and short grasses. Topography of the study area varied from nearly level plains to very gently sloping. The influence of climate, topography and vegetation acting on parent material over a period of time resulted in development of different soils viz., Entisols, Inceptisols and Vertisols in the study area.

\section{Soil taxonomy}

Based on the soil morphological, physical and physico-chemical properties, the typifying pedons were classified into the orders Entisols, Inceptisols and Vertisols according to Soil Taxonomy (Soil Survey Staff, 2014). Pedons 3 and 6 which do not have any diagnostic horizons were classified under Entisols. Pedons 1, 4, 5 and 7 which exhibited cambic $(\mathrm{Bw})$ sub-surface diagnostic horizon, were classified under Inceptisols. Pedon 2 was classified under Vertisols due to presence of vertic features such as slickensides, pressure faces, and cracks in the B horizon.

The pedons 1, 4, 5 and 7 were classified under Ustepts at sub-order level due to ustic soil moisture regime and under Haplustepts at great group level as they did not show either duripan or calcic horizon and the base saturation was more than $60 \%$ at a depth between 0.25 to $0.75 \mathrm{~m}$ from the surface. All these four pedons did not show lithic contact with in $50 \mathrm{~cm}$ from the soil surface or any vertic properties. Hence, these pedons were logically classified as Typic Haplustepts at sub-group level.

The pedon 2 was classified under usterts at sub-order level due to presence of ustic soil moisture regime. At great group level, it was classified under Haplusterts due to the absence of salic, gypsic, calcic or petro calcic horizons within $100 \mathrm{~cm}$ of mineral soil surface. 
Int.J.Curr.Microbiol.App.Sci (2017) 6(10): 2699-2712

Table.1 Landscape characteristics of pedons

\begin{tabular}{|c|c|c|c|c|c|c|}
\hline Pedons / Villages & Location & Elevation above mean sea level (m) & Physiography & Slope $(\%)$ & Drainage & Parent material \\
\hline P1 Gundlasamudram & $\begin{array}{l}15^{\circ} 34^{\prime} 5739^{\prime \prime} \mathrm{N} \\
79^{\circ} 36^{\prime} 5410^{\prime \prime} \mathrm{E}\end{array}$ & 94 & Uplands & $1-3$ & Well drained & $\begin{array}{c}\text { Weathered calcareous } \\
\text { gneiss }\end{array}$ \\
\hline P2 Vallaipalem & $\begin{array}{l}15^{\circ} 28^{\prime} 5758^{\prime \prime} \mathrm{N} \\
79^{\circ} 45^{\prime} 4394^{\prime \prime} \mathrm{E}\end{array}$ & 50 & Plains & $0-1$ & Imperfectly drained & Weathered granite-gneiss \\
\hline P3 Pedda alavalapadu & $\begin{array}{l}15^{\circ} 25^{\prime} 5891^{\prime \prime} \mathrm{N} \\
79^{\circ} 44^{\prime} 5437^{\prime \prime} \mathrm{E}\end{array}$ & 51 & Uplands & $1-3$ & Well drained & Granite-gneiss \\
\hline P4 Vijayalaxmipuram & $\begin{array}{l}15^{\circ} 27^{\prime} 1389^{\prime \prime} \mathrm{N} \\
79^{\circ} 41^{\prime 2} 2822^{\prime \prime} \mathrm{E}\end{array}$ & 70 & Uplands & $1-3$ & Well drained & Weathered granite-gneiss \\
\hline P5 Rajupalem & $\begin{array}{l}15^{\circ} 29^{\prime} 0298^{\prime \prime} \mathrm{N} \\
79^{\circ} 39^{\prime} 3908^{\prime \prime} \mathrm{E}\end{array}$ & 80 & Uplands & $1-3$ & Well drained & Weathered granite-gneiss \\
\hline P6 Gangapalem & $\begin{array}{l}15^{\circ} 39^{\prime} 0326^{\prime \prime} \mathrm{N} \\
79^{\circ} 45^{\prime} 4935^{\prime \prime} \mathrm{E}\end{array}$ & 77 & Uplands & $1-3$ & Well drained & Granite-gneiss \\
\hline P7 Bakkireddipalem & $\begin{array}{l}15^{\circ} 37^{\prime} 2292^{\prime \prime} \mathrm{N} \\
79^{\circ} 46^{\prime} 1078^{\prime \prime} \mathrm{E}\end{array}$ & 65 & Plains & $0-1$ & Moderately well drained & Weathered granite-gneiss \\
\hline
\end{tabular}

Table.2 Morphological characteristics of the soils

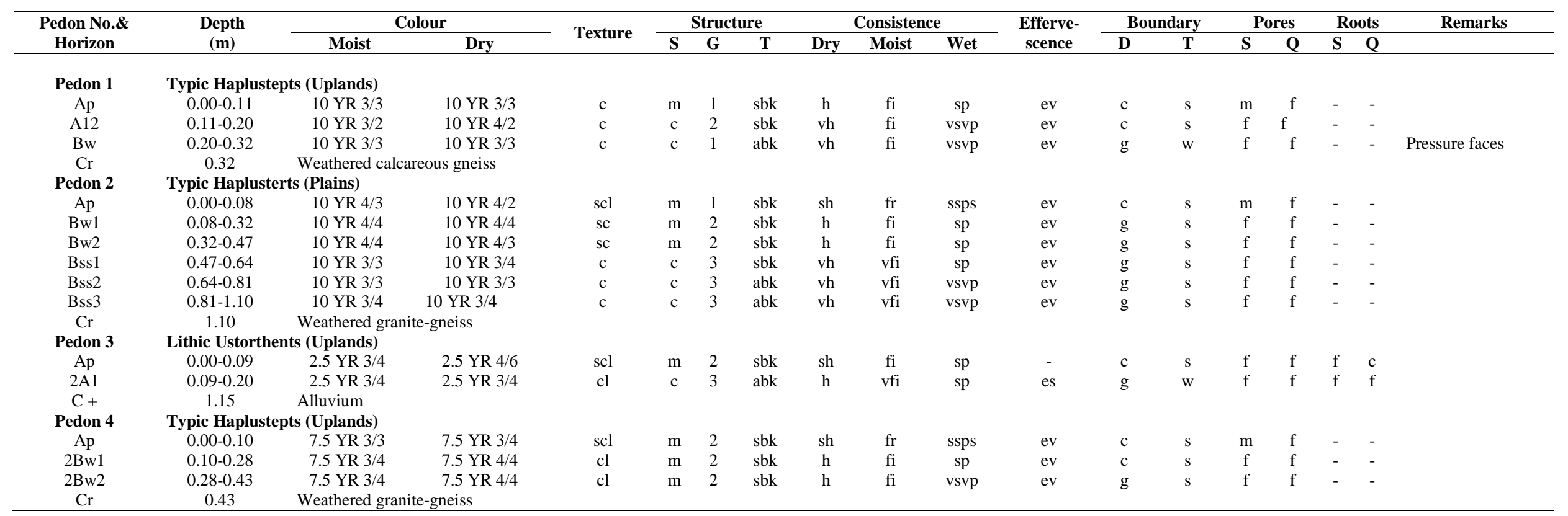


Table.2 (Contd.).

\begin{tabular}{|c|c|c|c|c|c|c|c|c|c|c|c|c|c|c|c|c|c|c|}
\hline \multirow{2}{*}{$\begin{array}{c}\text { Pedon } \\
\text { No.\& } \\
\text { Horizon }\end{array}$} & \multirow{2}{*}{$\begin{array}{c}\text { Depth } \\
\text { (m) }\end{array}$} & \multicolumn{2}{|c|}{ Colour } & \multirow{2}{*}{ Texture } & \multicolumn{3}{|c|}{ Structure } & \multicolumn{3}{|c|}{ Consistence } & \multirow{2}{*}{$\begin{array}{c}\text { Effer- } \\
\text { vescence }\end{array}$} & \multicolumn{2}{|c|}{ Boundary } & \multicolumn{2}{|c|}{ Pores } & \multicolumn{2}{|c|}{ Roots } & \multirow[t]{2}{*}{ Remarks } \\
\hline & & Moist & Dry & & $\mathbf{S}$ & $\mathbf{G}$ & $\mathbf{T}$ & Dry & Moist & Wet & & D & $\mathbf{T}$ & $\mathbf{S}$ & $\mathbf{Q}$ & $\mathbf{S}$ & $\mathbf{Q}$ & \\
\hline Pedon 5 & \multicolumn{3}{|c|}{ Typic Haplustepts (Uplands) } & & & & & & & & & & & & & & & \\
\hline Ap & $0.00-0.13$ & $7.5 \mathrm{YR} 3 / 4$ & $7.5 \mathrm{YR} 3 / 4$ & $\mathrm{sl}$ & $\mathrm{f}$ & 1 & sbk & $\mathrm{s}$ & $\mathrm{fr}$ & ssps & ev & $\mathrm{c}$ & $\mathrm{s}$ & $\mathrm{c}$ & $\mathrm{f}$ & $\mathrm{f}$ & $\mathrm{c}$ & \\
\hline $2 \mathrm{Bw}$ & $0.13-0.33$ & 7.5 YR $3 / 3$ & 7.5 YR 3/4 & $\mathrm{scl}$ & $\mathrm{m}$ & 2 & sbk & $\mathrm{sh}$ & $\mathrm{fr}$ & $\mathrm{sp}$ & ev & $\mathrm{g}$ & $\mathrm{s}$ & $\mathrm{f}$ & $\mathrm{f}$ & $\mathrm{f}$ & $\mathrm{f}$ & \\
\hline $\mathrm{Cr}$ & 0.33 & \multicolumn{2}{|c|}{ Weathered granite-gneiss } & & & & & & & & & & & & & & & \\
\hline Pedon 6 & \multicolumn{3}{|c|}{ Lithic Ustorthents (Uplands) } & & & & & & & & & & & & & & & \\
\hline Ap & $0.00-0.10$ & $10 \mathrm{YR} 3 / 2$ & $10 \mathrm{YR} 4 / 2$ & $\mathrm{scl}$ & $\mathrm{m}$ & 2 & sbk & $\mathrm{sh}$ & $\mathrm{fr}$ & ssps & ev & $\mathrm{c}$ & $\mathrm{s}$ & $\mathrm{f}$ & $\mathrm{m}$ & $\mathrm{f}$ & $\mathrm{c}$ & \\
\hline A1 & $0.10-0.20$ & $10 \mathrm{YR} 3 / 2$ & $10 \mathrm{YR} 4 / 3$ & $\mathrm{scl}$ & $\mathrm{m}$ & 2 & sbk & $\mathrm{sh}$ & $\mathrm{fr}$ & ssps & ev & $\mathrm{c}$ & $\mathrm{s}$ & $\mathrm{f}$ & $\mathrm{f}$ & $\mathrm{f}$ & $\mathrm{f}$ & \\
\hline $\mathrm{R}$ & 0.20 & \multicolumn{2}{|l|}{ Granite-gneiss } & & & & & & & & & & & & & & & \\
\hline Pedon 7 & \multicolumn{3}{|c|}{ Typic Haplustepts (Plains) } & & & & & & & & & & & & & & & \\
\hline Ap & $0.00-0.10$ & $5 \mathrm{YR} 3 / 4$ & $5 \mathrm{YR} 4 / 4$ & $\mathrm{scl}$ & $\mathrm{f}$ & 1 & sbk & $\mathrm{sh}$ & fr & ssps & es & $\mathrm{c}$ & $\mathrm{s}$ & $\mathrm{f}$ & $\mathrm{f}$ & $\mathrm{f}$ & $\mathrm{f}$ & \\
\hline Bw1 & $0.10-0.26$ & $5 \mathrm{YR} 3 / 3$ & $5 \mathrm{YR} 4 / 3$ & $\mathrm{scl}$ & $\mathrm{f}$ & 2 & sbk & $\mathrm{sh}$ & $\mathrm{fr}$ & ssps & es & $\mathrm{g}$ & $\mathrm{s}$ & $\mathrm{f}$ & $\mathrm{f}$ & $\mathrm{f}$ & $\mathrm{f}$ & \\
\hline Bw2 & $0.26-0.46$ & $5 \mathrm{YR} 3 / 3$ & $5 \mathrm{YR} 4 / 3$ & $\mathrm{sc}$ & $\mathrm{f}$ & 2 & sbk & $\mathrm{h}$ & fr & $\mathrm{sp}$ & es & $\mathrm{g}$ & $\mathrm{s}$ & $\mathrm{f}$ & $\mathrm{f}$ & $\mathrm{f}$ & $\mathrm{f}$ & \\
\hline $\mathrm{Cr}$ & 0.46 & \multicolumn{2}{|c|}{ Weathered granite-gneiss } & & & & & & & & & & & & & & & \\
\hline
\end{tabular}

Texture: $\mathrm{c}$ - clay, cl - clay loam, l - loam, s - sand, sl - sandy loam, scl - sandy clay loam, sc - sandy clay, ls - loamy sand

Structure: Size (S) - vf - very fine, f - fine, $\mathrm{m}$ - medium, c - coarse; Grade $(\mathrm{G})$ - O - structureless, 1 - weak, 2 - moderate, 3 - strong; Type (T) cr - crumb, sg single grain, abk - angular blocky, sbk - sub-angular blocky. Consistence:

Dry: $\mathrm{s}$ - soft, $\mathrm{l}$ - loose, sh - slightly hard, h - hard, vh - very hard

Moist: 1 - loose, fr - friable, fi - firm, vfi - very firm

Wet: so - non-sticky, ss - slightly sticky, s - sticky, vs - very sticky; po - non-plastic, ps - slightly plastic, p - plastic, vp - very plastic

Cutans: Ty - type $-\mathrm{t}-$ Argillan, Th - Thickness, $\mathrm{tn}-$ thin, th - thick, Quantity (Q), p - patchy, $\mathrm{c}-$ continuous

Pores: Size (S) f - fine, $\mathrm{m}$ - medium, c- coarse; $\mathrm{Q}$ - Quantity, $\mathrm{f}$ - few, c - common, $\mathrm{m}$ - many

Roots: Size (S) f - fine, m- medium, c- coarse; Q - Quantity, f - few, c - common, $m$ - many

Effervescence: es - strong effervescence, ev - violent effervescence

Boundary: D - Distinctness, c - clear, g - gradual, $\mathrm{d}$ - diffuse

$\mathrm{T}$ - Topography; $\mathrm{s}$ - smooth; $\mathrm{w}$ - wavy 
Int.J.Curr.Microbiol.App.Sci (2017) 6(10): 2699-2712

Table.3 Physical characteristics of the soils

\begin{tabular}{|c|c|c|c|c|c|c|c|}
\hline $\begin{array}{l}\text { Pedon No. } \\
\& \\
\text { Horizon }\end{array}$ & $\begin{array}{c}\text { Depth } \\
(\mathrm{m})\end{array}$ & $\begin{array}{c}\text { Sand } \\
(\%) \\
(0.05-2.0 \\
\mathrm{mm})\end{array}$ & $\begin{array}{c}\text { Silt } \\
(\%) \\
(0.002-0.05 \\
\mathrm{mm})\end{array}$ & $\begin{array}{c}\text { Clay } \\
(\%) \\
(<0.002 \mathrm{~mm})\end{array}$ & $\begin{array}{c}\text { Bulk } \\
\text { density } \\
\left(\mathrm{Mg} \mathrm{m}^{-3}\right)\end{array}$ & $\begin{array}{l}\text { Particle } \\
\text { density } \\
\left(\mathrm{Mg} \mathrm{m}^{-3}\right)\end{array}$ & $\begin{array}{l}\text { Water holding } \\
\text { capacity }(\%)\end{array}$ \\
\hline Pedon 1 & \multicolumn{7}{|c|}{ Typic Haplustepts (Uplands) } \\
\hline Ap & $0.00-0.11$ & 37.79 & 15.12 & 47.09 & 1.15 & 2.37 & 35.46 \\
\hline A12 & $0.11-0.20$ & 37.99 & 19.15 & 42.86 & 1.19 & 2.41 & 33.31 \\
\hline $\mathrm{Bw}$ & $0.20-0.32$ & 37.72 & 13.02 & 49.26 & 1.34 & 2.43 & 43.83 \\
\hline $\mathrm{Cr}$ & 0.32 & \multicolumn{6}{|c|}{ Weathered calcareous gneiss } \\
\hline Pedon 2 & \multicolumn{7}{|c|}{ Typic Haplusterts (Plains) } \\
\hline Ap & $0.00-0.08$ & 50.70 & 14.52 & 34.78 & 1.36 & 2.35 & 32.80 \\
\hline Bw1 & $0.08-0.32$ & 48.11 & 13.04 & 38.85 & 1.37 & 2.41 & 36.33 \\
\hline $\mathrm{Bw} 2$ & $0.32-0.47$ & 48.52 & 11.28 & 43.20 & 1.43 & 2.47 & 42.62 \\
\hline Bss1 & $0.47-0.64$ & 41.32 & 13.20 & 45.48 & 1.42 & 2.52 & 39.24 \\
\hline Bss2 & $0.64-0.81$ & 36.17 & 16.55 & 47.28 & 1.47 & 2.52 & 46.38 \\
\hline Bss3 & $0.81-1.10$ & 35.26 & 17.73 & 47.01 & 1.52 & 2.56 & 46.60 \\
\hline $\mathrm{Cr}$ & 1.10 & \multicolumn{6}{|c|}{ Weathered granite-gneiss } \\
\hline Pedon 3 & \multicolumn{7}{|c|}{ Lithic Ustorthents (Uplands) } \\
\hline Ap & $0.00-0.09$ & 55.96 & 9.74 & 34.30 & 1.43 & 2.49 & 23.36 \\
\hline $2 \mathrm{~A} 1$ & $0.09-0.20$ & 40.33 & 23.32 & 36.35 & 1.51 & 2.50 & 29.27 \\
\hline $\mathrm{R}$ & 0.20 & \multicolumn{6}{|c|}{ Granite-gneiss } \\
\hline Pedon 4 & \multicolumn{7}{|c|}{ Typic Haplustepts (Uplands) } \\
\hline Ap & $0.00-0.10$ & 62.61 & 11.54 & 25.85 & 1.46 & 2.52 & 23.17 \\
\hline 2Bw1 & $0.10-0.28$ & 44.74 & 20.12 & 35.14 & 1.48 & 2.51 & 26.74 \\
\hline 2Bw2 & $0.28-0.43$ & 41.07 & 22.62 & 36.31 & 1.52 & 2.54 & 30.15 \\
\hline $\mathrm{Cr}$ & 0.43 & \multicolumn{6}{|c|}{ Weathered granite-gneiss } \\
\hline Pedon 5 & \multicolumn{7}{|c|}{ Typic Haplustepts (Uplands) } \\
\hline Ap & $0.00-0.13$ & 77.33 & 6.85 & 15.82 & 1.47 & 2.56 & 18.54 \\
\hline $2 \mathrm{Bw}$ & $0.13-0.33$ & 60.48 & 14.75 & 24.77 & 1.51 & 2.53 & 23.52 \\
\hline $\mathrm{Cr}$ & 0.33 & \multicolumn{6}{|c|}{ Weathered granite-gneiss } \\
\hline Pedon 6 & \multicolumn{7}{|c|}{ Lithic Ustorthents (Uplands) } \\
\hline Ap & $0.00-0.10$ & 64.35 & 9.14 & 26.51 & 1.57 & 2.48 & 22.54 \\
\hline A1 & $0.10-0.20$ & 61.16 & 9.48 & 29.36 & 1.66 & 2.47 & 24.66 \\
\hline $\mathrm{R}$ & 0.20 & \multicolumn{6}{|c|}{ Granite-gneiss } \\
\hline Pedon 7 & \multicolumn{7}{|c|}{ Typic Haplustepts (Plains) } \\
\hline Ap & $0.00-0.10$ & 68.78 & 7.65 & 23.57 & 1.69 & 2.67 & 24.89 \\
\hline Bw1 & $0.10-0.26$ & 58.38 & 8.68 & 32.94 & 1.76 & 2.61 & 26.75 \\
\hline Bw2 & $0.26-0.46$ & 51.94 & 9.17 & 38.89 & 1.79 & 2.68 & 31.54 \\
\hline $\mathrm{Cr}$ & 0.46 & \multicolumn{6}{|c|}{ Weathered granite-gneiss } \\
\hline
\end{tabular}


Table.4 Physico-chemical properties of the soils

\begin{tabular}{|c|c|c|c|c|c|c|c|c|c|c|c|}
\hline \multirow{2}{*}{$\begin{array}{l}\text { Pedon } \\
\text { No. \& } \\
\text { Horizon }\end{array}$} & \multirow[t]{2}{*}{$\begin{array}{l}\text { Depth } \\
(\mathrm{m})\end{array}$} & \multirow[t]{2}{*}{$\underset{(1: 2.5)}{\mathrm{pH}}$} & \multirow[t]{2}{*}{$\begin{array}{c}\mathrm{EC} \\
\left(\mathrm{dsm}^{-1}\right)\end{array}$} & \multirow{2}{*}{$\begin{array}{l}\text { Organic } \\
\text { carbon } \\
\left(\mathrm{g} \mathrm{kg}^{-1}\right)\end{array}$} & \multirow[t]{2}{*}{$\begin{array}{c}\mathrm{CaCO}_{3} \\
(\%)\end{array}$} & \multirow[t]{2}{*}{$\begin{array}{l}\mathrm{CEC} \\
(\mathrm{cmol} \\
\left(\mathrm{p}^{+}\right) \\
\left.\mathrm{kg}^{-1}\right)\end{array}$} & \multicolumn{4}{|c|}{ 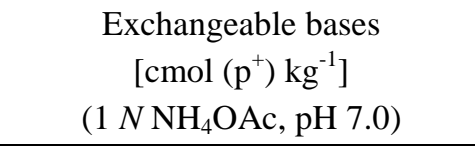 } & \multirow[t]{2}{*}{$\begin{array}{c}\text { Base } \\
\text { saturatior } \\
(\%)\end{array}$} \\
\hline & & & & & & & $\mathrm{Ca}^{2+}$ & $\mathrm{Mg}^{2+}$ & $\mathrm{Na}^{+}$ & $\mathrm{K}^{+}$ & \\
\hline \multicolumn{12}{|c|}{ Pedon 1. Typic Haplustepts (Uplands) } \\
\hline Ap & $0.00-0.11$ & 9.35 & 0.06 & 0.65 & 10.54 & 50.12 & 31.05 & 10.95 & 7.36 & 0.78 & 86.07 \\
\hline A12 & $0.11-0.20$ & 9.53 & 0.06 & 0.58 & 10.90 & 52.09 & 32.40 & 10.75 & 7.56 & 0.63 & 87.04 \\
\hline $\mathrm{Bw}$ & $0.20-0.32$ & 9.56 & 0.05 & 0.39 & 11.86 & 53.85 & 33.05 & 11.00 & 7.82 & 0.50 & 86.41 \\
\hline $\mathrm{Cr}$ & 0.32 & \multicolumn{10}{|c|}{ Weathered calcareous gneiss } \\
\hline \multicolumn{12}{|c|}{ Pedon 2. Typic Haplusterts (Plains) } \\
\hline Ap & $0.00-0.08$ & 9.86 & 0.82 & 0.36 & 10.37 & 14.35 & 6.05 & 2.21 & 0.56 & 0.07 & 61.95 \\
\hline Bw1 & $0.08-0.32$ & 9.88 & 1.32 & 0.26 & 11.16 & 31.72 & 14.10 & 4.35 & 1.42 & 0.18 & 63.21 \\
\hline Bw2 & $0.32-0.47$ & 9.86 & 1.32 & 0.22 & 11.34 & 40.37 & 22.85 & 2.05 & 0.10 & 0.22 & 62.47 \\
\hline Bss1 & $0.47-0.64$ & 10.00 & 0.95 & 0.23 & 11.45 & 46.07 & 29.65 & 10.35 & 1.32 & 0.76 & 84.83 \\
\hline Bss2 & $0.64-0.81$ & 9.87 & 1.38 & 0.19 & 11.72 & 43.38 & 27.10 & 9.50 & 1.28 & 0.62 & 88.75 \\
\hline Bss3 & $0.81-1.10$ & 9.82 & 1.26 & 0.16 & 11.86 & 33.87 & 20.50 & 7.95 & 1.12 & 0.52 & 88.84 \\
\hline $\mathrm{Cr}$ & 1.10 & \multicolumn{10}{|c|}{ Weathered granite-gneiss } \\
\hline \multicolumn{12}{|c|}{ Pedon 3. Lithic Ustorthents (Uplands) } \\
\hline Ap & $0.00-0.09$ & 7.68 & 0.05 & 0.58 & 5.42 & 16.16 & 8.05 & 4.75 & 0.20 & 0.18 & 81.56 \\
\hline $2 \mathrm{~A} 1$ & $0.09-0.20$ & 7.86 & 0.06 & 0.43 & 6.86 & 36.58 & 24.45 & 7.90 & 1.36 & 0.23 & 92.78 \\
\hline $\mathrm{R}$ & 0.20 & \multicolumn{10}{|c|}{ Granite-gneiss } \\
\hline \multicolumn{12}{|c|}{ Pedon 4. Typic Haplustepts (Uplands) } \\
\hline Ap & $0.00-0.10$ & 10.48 & 0.04 & 0.58 & 14.52 & 10.05 & 5.65 & 2.15 & 0.44 & 0.34 & 85.37 \\
\hline 2Bw1 & $0.10-0.28$ & 10.44 & 0.04 & 0.39 & 15.26 & 14.49 & 7.30 & 3.90 & 0.42 & 0.32 & 82.40 \\
\hline $2 \mathrm{Bw} 2$ & $0.28-0.43$ & 10.09 & 1.04 & 0.34 & 17.64 & 16.26 & 7.55 & 4.85 & 0.40 & 0.27 & 80.38 \\
\hline $\mathrm{Cr}$ & 0.43 & \multicolumn{10}{|c|}{ Weathered granite-gneiss } \\
\hline \multicolumn{12}{|c|}{ Pedon 5. Typic Haplustepts (Uplands) } \\
\hline Ap & $0.00-0.13$ & 8.31 & 0.06 & 0.72 & 10.35 & 15.87 & 9.50 & 4.45 & 0.72 & 0.58 & 85.34 \\
\hline $2 \mathrm{Bw}$ & $0.13-0.33$ & 8.09 & 0.06 & 0.63 & 10.71 & 17.28 & 10.25 & 4.90 & 0.82 & 0.71 & 86.51 \\
\hline $\mathrm{Cr}$ & 0.33 & \multicolumn{10}{|c|}{ Weathered granite-gneiss } \\
\hline \multicolumn{12}{|c|}{ Pedon 6. Lithic Ustorthents (Uplands) } \\
\hline Ap & $0.00-0.10$ & 7.91 & 0.09 & 0.19 & 10.12 & 17.03 & 10.55 & 4.55 & 0.77 & 0.72 & 87.18 \\
\hline A1 & $0.10-0.20$ & 8.02 & 0.20 & 0.14 & 11.54 & 17.38 & 10.30 & 4.90 & 0.82 & 0.74 & 91.19 \\
\hline $\mathrm{R}$ & 0.20 & \multicolumn{10}{|c|}{ Granite-gneiss } \\
\hline \multicolumn{12}{|c|}{ Pedon 7. Typic Haplustepts (Plains) } \\
\hline Ap & $0.00-0.10$ & 7.65 & 0.27 & 0.26 & 8.66 & 10.05 & 5.65 & 2.15 & 0.42 & 0.37 & 85.47 \\
\hline Bw1 & $0.10-0.26$ & 7.63 & 0.22 & 0.21 & 7.53 & 9.14 & 4.85 & 1.35 & 0.66 & 0.52 & 80.74 \\
\hline Bw2 & $0.26-0.46$ & 7.92 & 0.36 & 0.18 & 8.92 & 28.12 & 12.35 & 4.80 & 0.92 & 0.34 & 65.47 \\
\hline $\mathrm{Cr}$ & 0.46 & \multicolumn{10}{|c|}{ Weathered granite-gneiss } \\
\hline
\end{tabular}


Table.5 Available macro and micro nutrients of the soils

\begin{tabular}{|c|c|c|c|c|c|c|c|c|c|}
\hline \multirow{3}{*}{$\begin{array}{l}\text { Pedon No. } \\
\& \text { Horizon }\end{array}$} & \multirow{3}{*}{$\begin{array}{l}\text { Depth } \\
(\mathrm{m})\end{array}$} & \multicolumn{4}{|c|}{ Available macronutrients } & \multicolumn{4}{|c|}{ Available micronutrients } \\
\hline & & $\mathrm{N}$ & $\mathrm{P}$ & $\mathrm{K}$ & \multirow{2}{*}{$\begin{array}{c}\mathrm{S} \\
\mathrm{mg} \mathrm{kg}^{-1}\end{array}$} & $\mathrm{Zn}$ & $\mathrm{Cu}$ & $\mathrm{Fe}$ & $\mathrm{Mn}$ \\
\hline & & \multicolumn{3}{|c|}{------- $\mathrm{kg} \mathrm{ha}^{-1}$} & & \multicolumn{4}{|c|}{ - } \\
\hline \multicolumn{10}{|c|}{ Pedon 1 Typic Haplustepts (Uplands) } \\
\hline Ap & $0.00-0.11$ & 88 & 17.20 & 315 & 27.21 & 0.92 & 0.72 & 6.32 & 12.30 \\
\hline A12 & $0.11-0.20$ & 75 & 15.68 & 309 & 23.52 & 0.55 & 0.81 & 4.06 & 11.83 \\
\hline $\mathrm{Bw}$ & $0.20-0.32$ & 88 & 16.18 & 334 & 18.24 & 0.43 & 0.70 & 5.24 & 9.76 \\
\hline $\mathrm{Cr}$ & 0.32 & \multicolumn{4}{|c|}{ Weathered calcareous gneiss } & & & & \\
\hline Pedon 2 & \multicolumn{5}{|c|}{ Typic Haplusterts (Plains) } & & & & \\
\hline Ap & $0.00-0.08$ & 88 & 15.32 & 166 & 20.42 & 0.48 & 0.72 & 9.12 & 9.88 \\
\hline Bw1 & $0.08-0.32$ & 75 & 13.24 & 149 & 17.52 & 0.39 & 0.69 & 8.34 & 11.20 \\
\hline $\mathrm{Bw} 2$ & $0.32-0.47$ & 75 & 14.56 & 214 & 14.74 & 0.48 & 0.74 & 8.15 & 12.73 \\
\hline Bss1 & $0.47-0.64$ & 63 & 16.32 & 259 & 12.56 & 0.31 & 0.79 & 7.48 & 14.91 \\
\hline Bss2 & $0.64-0.81$ & 88 & 15.34 & 391 & 10.08 & 0.49 & 0.86 & 8.07 & 10.56 \\
\hline Bss3 & $0.81-1.10$ & 63 & 17.47 & 379 & 12.35 & 0.13 & 1.26 & 7.48 & 9.32 \\
\hline $\mathrm{Cr}$ & 1.10 & \multicolumn{4}{|c|}{ Weathered granite-gneiss } & & & & \\
\hline Pedon 3 & \multicolumn{5}{|c|}{ Lithic Ustorthents (Uplands) } & & & & \\
\hline Ap & $0.00-0.09$ & 88 & 15.65 & 257 & 16.03 & 0.64 & 0.61 & 6.19 & 3.88 \\
\hline $2 \mathrm{~A} 1$ & $0.09-0.20$ & 188 & 21.62 & 161 & 12.53 & 0.60 & 0.34 & 5.82 & 2.92 \\
\hline $\mathrm{R}$ & 0.20 & \multicolumn{4}{|c|}{ Granite-gneiss } & & & & \\
\hline Pedon 4 & \multicolumn{5}{|c|}{ Typic Haplustepts (Uplands) } & & & & \\
\hline Ap & $0.00-0.10$ & 251 & 17.69 & 158 & 31.32 & 0.59 & 1.06 & 7.40 & 10.55 \\
\hline 2Bw1 & $0.10-0.28$ & 113 & 16.63 & 170 & 28.31 & 0.52 & 1.26 & 7.22 & 6.45 \\
\hline 2Bw2 & $0.28-0.43$ & 75 & 15.84 & 137 & 23.68 & 0.41 & 1.92 & 6.73 & 5.26 \\
\hline $\mathrm{Cr}$ & 0.43 & \multicolumn{4}{|c|}{ Weathered granite-gneiss } & & & & \\
\hline Pedon 5 & \multicolumn{5}{|c|}{ Typic Haplustepts (Uplands) } & & & & \\
\hline Ap & $0.00-0.13$ & 138 & 14.25 & 173 & 18.83 & 0.65 & 1.21 & 6.04 & 8.44 \\
\hline $2 \mathrm{Bw}$ & $0.13-0.33$ & 113 & 15.36 & 140 & 14.35 & 0.63 & 1.35 & 5.69 & 7.23 \\
\hline $\mathrm{Cr}$ & 0.33 & \multicolumn{4}{|c|}{ Weathered granite-gneiss } & & & & \\
\hline Pedon 6 & \multicolumn{5}{|c|}{ Lithic Ustorthents (Uplands) } & & & & \\
\hline Ap & $0.00-0.10$ & 188 & 24.03 & 166 & 14.08 & 0.93 & 1.75 & 9.92 & 17.60 \\
\hline A1 & $0.10-0.20$ & 176 & 18.71 & 139 & 12.36 & 0.88 & 1.70 & 7.86 & 13.85 \\
\hline $\mathrm{R}$ & 0.20 & \multicolumn{4}{|c|}{ Granite-gneiss } & & & & \\
\hline Pedon 7 & \multicolumn{5}{|c|}{ Typic Haplustepts (Plains) } & & & & \\
\hline Ap & $0.00-0.10$ & 188 & 21.42 & 286 & 13.58 & 0.64 & 1.86 & 16.52 & 21.16 \\
\hline Bw1 & $0.10-0.26$ & 163 & 17.84 & 258 & 11.23 & 0.62 & 1.82 & 12.82 & 18.16 \\
\hline Bw2 & $0.26-0.46$ & 125 & 18.36 & 224 & 9.62 & 0.47 & 1.54 & 12.48 & 16.54 \\
\hline $\mathrm{Cr}$ & 0.46 & \multicolumn{4}{|c|}{ Weathered granite-gneiss } & & & & \\
\hline
\end{tabular}


The pedons 3 and 6 were placed under Psamments at sub-order level because of high sand fraction, absence of any diagnostic horizon and not showing permanent saturation with water. These were grouped under Ustipsamments at great group level due to the presence of ustic soil moisture regime. Finally, they are classified under Typic Ustipsamments at sub-group level as they showed typical characteristics of Ustipsamments.

\section{Soil fertility}

Soil fertility indicates the status of amount and availability of essential nutrients in different soils with regard to plant growth.

\section{Macronutrients}

The available $\mathrm{N}$ content in the study area varied from 63 to $251 \mathrm{~kg} \mathrm{ha}^{-1}$ (Table 5) throughout the depth. Available nitrogen content was found to be maximum in surface horizons and decreased regularly with depth which is due to decreasing trend of organic carbon with depth and cultivation of crops are mainly confined to the surface horizon (rhizosphere) only and also, the depleted nitrogen from soils is supplemented by external addition of fertilizers at regular intervals during crop cultivation (Satish Kumar and Naidu, 2012).

The available $\mathrm{P}$ content ranged from 13.24 to $24.03 \mathrm{~kg} \mathrm{ha}^{-1}$ in soils of the study area. The highest available phosphorus was found in the surface horizons and decreased with depth, which might be due to the confinement of crop cultivation to the rhizosphere and supplementing the depleted phosphorus by external fertilizers and presence of free iron oxide and exchangeable $\mathrm{Al}^{3+}$ in smaller amounts (Thangasamy et al., 2005). The relatively low phosphorus contents in subsurface horizons were due to the fixation of released phosphorus by clay minerals and oxides of iron and aluminum.

The available $\mathrm{K}$ content of soils in the study area ranged from 137 to $391 \mathrm{~kg} \mathrm{ha}^{-1}$. The highest available potassium was found in the surface horizons and showed more or less a decreasing trend with depth, which might be attributed to more intense weathering, release of labile $\mathrm{K}$ from organic residues, upward translocation of potassium from lower depths along with capillary raise of ground water and application of K fertilizers (Sharma and Anil Kumar, 2003). The available sulphur in soils varied from 9.62 to $31.32 \mathrm{mg} \mathrm{kg}$. The available sulphur was found to be more in the surface horizons than sub-surface horizons due to higher amounts of organic matter in surface layers.

\section{Micronutrients}

The DTPA-extractable $\mathrm{Zn}$ in the study area ranged from 0.13 to $0.93 \mathrm{mg} \mathrm{kg}{ }^{-1}$ soil. Considering $0.6 \mathrm{mg} \mathrm{kg}^{-1}$ soil (Lindsay and Norvell, 1978) as critical level for available zinc, the pedons 2 and 4 were found to be deficient in available zinc throughout their depth. All other pedons were found to be sufficient in available $\mathrm{Zn}$ content (except in the $\mathrm{A} 12$ and $\mathrm{Bw}$ horizons of pedon 1 and $\mathrm{Bw} 2$ horizon of pedon 7). The low available zinc was due to calcareousness, high $\mathrm{pH}$ and low organic matter which have resulted in the formation of insoluble compounds of zinc or insoluble calcium zincate (Rattan and Sharma, 2004).

The DTPA-extractable Fe content varied from 4.06 to $16.52 \mathrm{mg} \mathrm{kg}^{-1}$ soil. According to the critical limit of $4.5 \mathrm{mg} \mathrm{kg}^{-1}$ soil, as given by Lindsay and Norvell (1978), the soils were found sufficient in available iron content. The distribution of available iron in general did not follow any definite pattern. The surface horizons contained more available $\mathrm{Fe}$ than 
sub-surface horizons in all the pedons in the study area, which is due to accumulation of organic carbon in the surface horizons. The organic carbon, due to its affinity to influence the solubility and availability of iron by chelation effect, might have protected the iron from oxidation and precipitation, which consequently increased the availability of iron. The low iron content in sub-surface horizons might be due to precipitation of $\mathrm{Fe}^{+2}$ by calcium carbonate concretions in calcareous soils and higher $\mathrm{pH}$ of these soils, which might have decreased the availability of Fe (Vijaya Kumar et al., 2013).

The DTPA-extractable copper (0.34 to 1.92 $\mathrm{mg} \mathrm{kg}{ }^{-1}$ ) and manganese (2.92 to $21.16 \mathrm{mg}$ $\mathrm{kg}^{-1}$ ) were found to be sufficient in all the soils of the study area as these nutrients are well above their critical limits of 0.2 and 1.0 $\mathrm{mg} \mathrm{kg}{ }^{-1}$, respectively (Lindsay and Norvell, 1978). The higher concentrations of available copper and manganese in these soils might be due to higher biological activity and the chelating effect of organic compounds that are released during the decomposition of organic matter left after crop harvesting (Verma et al., 2005).

Soils in the study area from central parts of Prakasam district were shallow to very deep in depth, weakly alkaline to alkali in reaction, non-saline and low to medium in organic carbon and the exchangeable complex was dominated by $\mathrm{Ca}^{2+}$ followed by $\mathrm{Mg}^{2+}, \mathrm{Na}^{+}$ and $\mathrm{K}^{+}$, respectively. The soils were low in available nitrogen, medium in available phosphorus, medium to high in available potassium and sufficient in available sulphur. However, soils were deficient in available zinc (except in the pedons 2 and 4 and in the subsurface horizons of pedon 1), and sufficient in available iron, copper and manganese. The soils of the study area were classified as Lithic Ustorthents (pedons 3 and 6), Typic Haplustepts (pedons 1, 4, 5 and 7) and Typic Haplusterts (pedon 2). The present study in the central parts of Prakasam district revealed that soil test based judicious application of organic materials in combination with chemical fertilizers to these soils not only helps in achieving sustainable higher yields in different crops, but also sustains the soil productivity for future generations without deteriorating soil health.

\section{References}

Ahuja, L.R., Naney, J., Williams, W.R.D. and Ross, J.R. 1988. Vertical variability of soil properties in a small watershed. Journal of Hydrology 99, 307-308.

AIS \& LUS 1970. Soil Survey Manual, All India Soil and Land Use Survey Organisation, IARI, New Delhi, pp. 163.

Ashokkumar, H.P., and Jagdish Prasad. 2010. Some typical sugarcane growing soils of Ahmadnagar district of Maharashtra. Their characterization, classification and nutritional status of soils and plants. Journal of the Indian Society of Soil Science 58, 257-266.

Bhaskar, B.P., Mishra, J.P., Utpal Baruah, Vadivelu, S., Sen, T.K., Butte, P.S. and Dutta, D.P. 2004. Soils on Jhum cultivated hill slopes of NarangKongripara watershed in Maghalaya. Journal of the Indian Society of Soil Science 52, 125-133.

Coughlan, K.J., Mcgarry, D. and Smith, G.D. 1986. The physical and mechanical characterization of Vertisols. In First Regional Seminar on Management of Vertisols under Semi-Arid Conditions. IBSRAM Proceeding Number 6, Nairobi, Kenya, pp. 89-106.

Geetha Sireesha, P.V., and Naidu, M.V.S. 2013. Studies on Genesis, Characterization and Classification of Soils in Semi-arid Agro-ecological Region: A Case Study in Banaganapalle 
Mandal of Kurnool District, Andhra Pradesh. Journal of the Indian Society of Soil Science. 61, 167-178.

Gurumurthy, P., Seshagiri Rao, M., Bhanu Prasad, V., Pillai, R.N and Lakshmi, G.V. 1996. Characterisation of red, black and associated soils of Giddalur mandal of Andhra Pradesh. The Andhra Agricultural Journal. 43, 123-127.

Leelavathi, G.P., Naidu, M.V.S., Ramavatharam, N and Karuna Sagar, G. 2009. Studies on genesis, classification and evaluation of soils for sustainable land use planning in Yerpedu mandal of Chittoor district, Andhra Pradesh. Journal of the Indian Society of Soil Science. 57, 109-120.

Leelavathi, G.P., Naidu, M.V.S., Ramavatharam, N and Karuna Sagar, G. 2010. Clay mineralogy of soils formed on granite-gneiss of Chittoor district, Andhra Pradesh. Journal of the Indian Society of Soil Science. 58, 376-383.

Lindsay, W.L., and Norvell, W.A. 1978. Development of DTPA soil test for zinc, iron, manganese and copper. Soil Science Society of America Journal 42, 421-428.

Manchanda, M.L., Kudrat, M. and Tiwari, A.K. 2002. Soil survey and mapping using remote sensing. Tropical Ecology. 43, 61-74

Murthy, A.S.P., 1988. Distribution, properties and management of Vertisols in India. Advances in Soil Science 8, 153-214.

Nayak, D.C., Sarkar, D. and Das, K. 2002. Forms and distribution of pedogenic iron, aluminum and manganese in some Benchmark soils of West Bengal. Journal of the Indian Society of Soil Science 50, 89-93.

Ramprakash, T., and Seshagiri Rao, M. 2002. Characterisation and classification of some soils in a part of Krishna district, Andhra Pradesh. The Andhra Agricultural Journal. 49, 228-236.
Sarkar, D., Gangopadhyay, S.K. and Velayutham, M. 2001. Soil toposequence relationship and classification in lower outlier of Chhotanagpur plateau. Agropedology 11, 29-36.

Satish Kumar, Y.S., and Naidu, M.V.S. 2012. Characteristics and classification of soils representing major landforms in Vadamalapeta mandal of Chittoor district, Andhra Pradesh. Journal of the Indian Society of Soil Science 60, 63-67. Shalima Devi, G.M., and Anil Kumar, K.S. 2010. Characterization and classification of coffee growing soils of Karnataka. Journal of the Indian Society of Soil Science. 58, 125-131.

Sharma, P.D., 2006. Soil Science research Vision 2025. Indian Society of Soil Science News Letter 20. P.1.

Sharma, S.S., Totawat, K.L. and Shyampura, R.L. 2004. Characterization and classification of salt-affected soils of southern Rajasthan. Journal of the Indian Society of Soil Science 52, 209213.

Sharma, V.K., and Anil Kumar. 2003. Characterization and classification of the soil of upper Maul Khad catchment in wet temperate zone of Himachal Pradesh. Agropedology 13, 39-49.

Simonson, R. W., 1959. Outline of a generalized theory of soil genesis. Soil Science Society of American Proceedings 23, 152-156.

Singh, I.S., and Agrawal, H.P. 2005. Characterization, genesis and classification of rice soils of eastern region of Varanasi, Uttar Pradesh. Agropedology 15, 29-38.

Soil Survey Division Staff. 2000. Soil Survey Manual (Indian Print), USDA Handbook 18, Us Govt. Printing Office, Washington.

Soil Survey Staff. 2014. Keys to soil taxonomy, $12^{\text {th }}$ edition, USDA, Natural 
Resource Conservation Service, Washington, DC.

Thangasamy, A., Naidu, M.V.S., Ramavatharam, N. and Raghava Reddy, C. 2005. Characterization, classification and evaluation of soil resources in Sivagiri micro - watershed of Chittoor district in Andhra Pradesh for sustainable land use planning. Journal of the Indian Society of Soil Science 53, 11-21.

Vara Prasad Rao, A.P., Naidu, M.V.S., Ramavatharam, N. and Rama Rao, G. 2008. Characterization, classification and evaluation of soils on different landforms in Ramachandrapuram mandal of Chittoor district in Andhra Pradesh for sustainable land use planning. Journal of the Indian Society of Soil Science 56, 23-33.

Verma, V.K., Setia, R.K., Sharma, P.K., Charanjit Singh and Ashok Kumar. 2005. Micronutrient distribution in soils developed on different physiographic units of Fatehgarh Sahib District of Punjab. Agropedology. 15, 70-75.

Vijaya Kumar, M., Lakshmi, G.V and Madhuvani, P. 2013. Appraisal of soil fertility in salt-affected soils of Ongole division, Prakasam district, Andhra Pradesh. Journal of the Indian Society of Soil Science 61, 333-340.

Walia, C.S., and Rao, Y.S. 1997. Characteristics and classification of some soils of Trans-Yamuna plains. Journal of the Indian Society of Soil Science 45, 156-162.

\section{How to cite this article:}

Chandra Sekhar, Ch., M.V.S. Naidu, T. Ramprakash and Balaguravaiah, D. 2017. Characterization and Classification of Soils in the Central Parts of Prakasam District in Andhra Pradesh, India. Int.J.Curr.Microbiol.App.Sci. 6(10): 2699-2712. doi: https://doi.org/10.20546/ijcmas.2017.610.318 